\title{
A dinâmica da Função de Reação do Banco Central do Brasil: uma possível fonte da perda de eficácia da política monetária
}

Rafael Quevedo do Amaral

\section{Introdução}

O regime de metas de inflação, adotado no Brasil a partir de junho de 1999, representou uma mudança na forma de condução da política monetária. Até então, a política macroeconômica era caracterizada pelo mecanismo de âncora cambial, na qual a taxa de câmbio era usada como instrumento na busca pela estabilidade de preços.

Esta nova arquitetura da política monetária foi introduzida com o objetivo de perseguir a estabilidade de preços mediante a utilização explícita de uma Regra de Taylor, o que parece ser uma característica particular do Brasil. Basicamente, o funcionamento do regime de metas de inflação passou a ocorrer da seguinte forma: o Banco Central fixa uma meta anual para a taxa de inflação, geralmente decrescente, e ajusta a taxa de juros sistematicamente de forma a alcançar seu objetivo.

Nesse novo processo de funcionamento da política monetária, os choques de demanda passam a ser inibidos mediante elevações da taxa Selic. A autoridade monetária adota um modelo de equações, das quais fazem parte uma Regra de Taylor, uma estimativa da taxa de inflação futura e uma função representativa do gap do produto. ${ }^{16}$ A partir da observação do comportamento das principais variáveis econômicas que compõe este modelo, são tomadas as decisões quanto à fixação da meta Selic. ${ }^{17}$

O objetivo deste artigo é estimar, a partir da utilização de um modelo de Vetores Auto-Regressivos $(\mathrm{VAR})^{18}$, a importância do canal do câmbio na função de reação do Banco Central. Para isso, procurar-se-á identificar a relação de causalidade entre as principais variáveis relevantes para a determinação da taxa de juros. As variáveis em consideração serão a taxa Selic, a taxa de câmbio, a taxa de utilização da capacidade produtiva na indústria, o Índice de Preços ao Consumidor Amplo (IPCA) e as expectativas quanto ao IPCA para 12 meses.

\footnotetext{
* Economista do Departamento Nacional de Produção Mineral, Ministério de Minas e Energia. Mestrando em Desenvolvimento Econômico pela UFPR. Endereço eletrônico: quevedoam@yahoo.com.br 16 Estimativa da diferença entre o produto efetivo e o potencial.

17 Para ter detalhes do modelo utilizado pelo Banco Central ver os Relatórios de Inflação (março e junho de 2002)

18 Para uma discussão da metodologia do modelo VAR, ver Amaral e Motta (2006).
} 


\section{Estimativa da dinâmica de determinação da taxa Selic}

A metodologia de Vetores Auto-Regressivos ${ }^{19}$ (VAR) aqui explorada, também é utilizada pelo Banco Central brasileiro nas estimativas a respeito das expectativas para o IPCA e para a produção industrial. Tal modelo é usado como forma de auxiliar nas decisões de política monetária. ${ }^{20}$

Também serão utilizados os seguintes testes: teste de Causalidade de Granger, decomposição da variância dos erros, teste de Johansen, teste de endogeneidade das variáveis (VAR Pairwise Granger Causality/Block Exogeneity Wald Tests) e teste de correlação serial dos resíduos. O método de Co-integração de Johansen foi escolhido por ser mais apropriado que o Teste de Engle Granger no caso de um VAR de mais de duas variáveis. ${ }^{21}$

Escolheu-se o nível de utilização da capacidade produtiva na indústria como uma proxy da relação produto efetivo/produto potencial. Para a estimação dos resultados, foi utilizado o software econométrico E-views, a partir do qual foram gerados os resultados apresentados no anexo.

Primeiramente, foram feitos os testes de estacionaridade das séries, considerando o nível de integração, defasagem da série e as especificações de intercepto e tendência, evitando problemas de espuriedade nos resultados, o que poderia levar a conclusões incorretas. Para tal procedimento, utilizou-se o teste de Dickey-Fuller Aumentado (ADF) ${ }^{22}$, que permite incorporar termos defasados extras da variável dependente eliminando o problema de autocorrelação dos resíduos. Os resultados encontrados estão resumidos na tabela 2 (Anexo).

$\mathrm{O}$ teste $\mathrm{ADF}$ indicou que todas as variáveis em consideração são estacionárias em $1^{\circ}$ diferença. Com exceção da taxa de câmbio, que apresentou uma defasagem de 6 períodos, as demais variáveis apresentaram 1 lag como a melhor defasagem pelo critério de Schwartz. A melhor defasagem para o modelo como um todo foi de 8 lags, sendo que para tal período o teste dos Multiplicadores de Lagrange (teste LM) não mostrou significância na presença de correlação serial dos resíduos, o que valida tal defasagem como a melhor escolha para o lag do modelo.

\footnotetext{
19 O termo auto-regressivo deve-se ao fato de utilizar a variável dependente de forma defasada como uma variável independente, enquanto o termo vetor se deve à utilização de um vetor de duas ou mais variáveis (ENDERS, 1995).

${ }^{20}$ Ver relatório de inflação de junho de 2004.

${ }^{21}$ Para uma discussão metodológica sobre os testes citados, ver Asterious (2006).

22 Também foi observado o teste de Phillip-Perron, que não mostrou divergência em relação ao ADF e, portanto, será desconsiderado para efeito de simplificação da análise.
} 
Com o valor da defasagem do sistema determinado, estimou-se o Teste de Causalidade de Granger, a decomposição da variância dos erros e a ordem de endogeneidade das variáveis - através do teste VAR Pairwise Granger Causality, conforme dispostos nas tabelas 5 e 7 , respectivamente.

Por fim, com o objetivo de verificar a existência de uma relação de longo prazo entre as variáveis, foi estimado o Teste de Co-integração de Johansen, onde se identificou a existência de pelo menos um vetor de co-integração. A importância do teste de co-integração advém do fato de que a diferenciação das variáveis leva a perdas nas propriedades de longo prazo, já que o modelo não tem uma solução de longo prazo (ASTERIOUS, 2006). A noção de co-integração, por sua vez, denota a possibilidade de combinar séries integradas de mesma ordem em uma série única que seja não estacionária.

A partir dos testes econométricos especificados, cujos resultados encontram-se em anexo, podem-se fazer algumas inferências sobre a interação dinâmica das variáveis em consideração. Para isso, o diagrama de causalidade entre as variáveis é um instrumento ilustrativo, já que resume as relações significativas do Teste de Causalidade de Granger.

A análise do Teste de Causalidade de Granger explicita os seguintes resultados: a Selic é causada pelas variáveis taxa de câmbio, IPCA e expectativas; o IPCA é causado pelas expectativas, pela taxa Selic e pela taxa de câmbio; as expectativas são causadas pela taxa de câmbio e o nível de utilização da capacidade é causado pela Selic. O seguinte diagrama de causalidade ilustra as relações entre as variáveis:

Figura 1. Diagrama de causalidade entre as variáveis

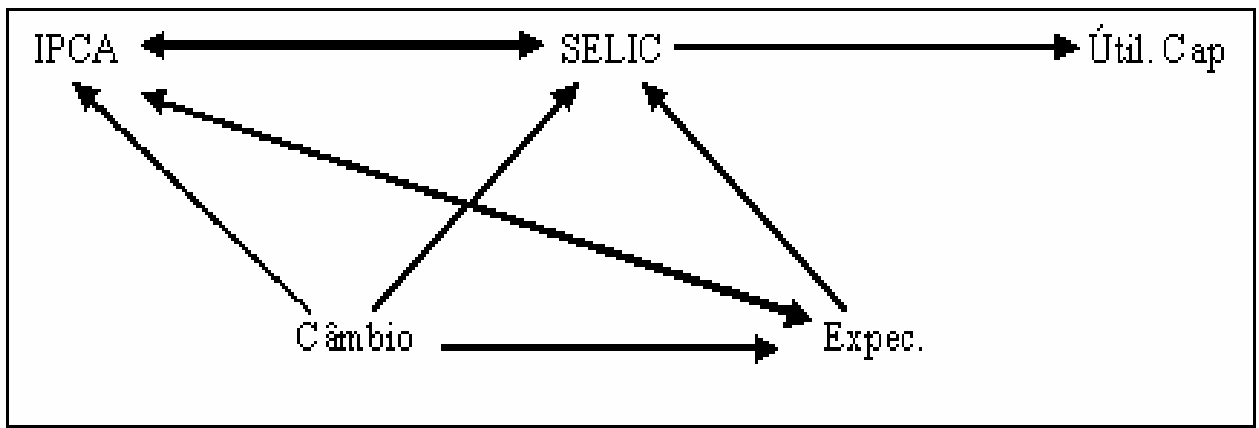

Observando-se as relações de causalidade, pode-se constatar que a taxa de câmbio é a principal variável de precedência, o que é confirmado pelo teste de endogeneidade das variáveis (VAR Paimvise Granger Causality/Block Exogeneity Wald Tests). Como pode ser constatado do teste de endogeneidade, as expectativas, o IPCA, a taxa Selic e o nível de 
utilização da capacidade seguem, nessa mesma ordem, a taxa de câmbio em grau decrescente de exogeneidade.

Dessa constatação, pode-se afirmar que a taxa de câmbio determina tanto diretamente, como indiretamente, via expectativas e IPCA, a taxa Selic. Ao determinar a taxa Selic por esses dois canais, essa última responde ao IPCA, determinando leve queda no nível de utilização da capacidade. Como pode ser observado nos gráficos de impulso-resposta (Figura 2), o impacto da taxa Selic sobre o IPCA e o nível de utilização da capacidade produtiva é consideravelmente modesto, o que pode estar implicando na necessidade de se utilizar "doses elevadas de juros" para conter a demanda agregada e a taxa de inflação.

Da decomposição da variância (Tabela 6), pode-se constatar que com uma defasagem de 7 meses, aproximadamente $65,5 \%$ da variação das expectativas ocorrem devido à variação cambial. No mesmo sentido, a variação cambial e as expectativas explicam, considerando uma defasagem de 8 e 2 meses, respectivamente, aproximadamente $46 \%$ e $43 \%$ da variação do IPCA. Ainda, com uma defasagem de 12 meses, aproximadamente $57 \%$ da variação da taxa Selic pode ser explicada pela variação da taxa de câmbio. Com 7 meses de defasagem, a variação da Selic explica diretamente somente 7,5\% da variação do IPCA. Por fim, adotando-se uma defasagem de 9 meses, algo em torno de $20 \%$ da variação da utilização da capacidade, a variável mais endógena do sistema, pode ser explicada pela variação da Selic.

\section{Conclusões}

Dessas relações apresentadas, podem-se inferir alguns resultados relevantes. A taxa de câmbio é a variável mais importante, em termos de causalidade, no sistema. Variações cambiais impactam a taxa Selic e o IPCA direta e indiretamente, mostrando-se a variável mais significativa na determinação da Regra de Taylor utilizada pelo Banco Central. Nesse contexto, a enorme participação dos preços administrados por contrato no IPCA - em torno de 30\% do índice - e sua grande sensibilidade ao câmbio, já que os mesmos são determinados pelo IGP-M e IGP-DI, parece ser um dos fatores explicativos da exagerada dependência da política monetária ao câmbio.

Apesar de a política monetária ter um caráter passivo dentro do sistema, ou seja, causar somente a taxa de inflação, o grau com que tal causalidade ocorre é extremamente modesto. Sendo assim, elevações da taxa Selic, tanto por demorarem em torno de um semestre para exercerem impacto negativo sobre os preços, como por não explicarem mais do que $8 \%$ da variação da taxa de inflação, mostram que esta variável está operando com 
considerável perda de eficácia. Além disso, os impactos da taxa de juros sobre o nível de utilização da capacidade produtiva não são revertidos nos preços, o que nos leva a crer que a autoridade monetária está respondendo a uma inflação primordialmente de custos, via redução das margens de lucro.

Pode-se inferir que tal anomalia na dinâmica da política monetária brasileira seja derivada tanto da participação dos preços administrados na formação do IPCA, como da peculiaridade do mercado de dívida pública no Brasil, onde o efeito riqueza da política monetária é inibido pela excessiva participação de títulos pós-fixados, mais especificamente Letras Financeiras do Tesouro (LFTs), na composição da Dívida Mobiliária Federal Interna $(\mathrm{DMFi}){ }^{23}$

\section{Anexos}

Tabela 1. Teste ADF

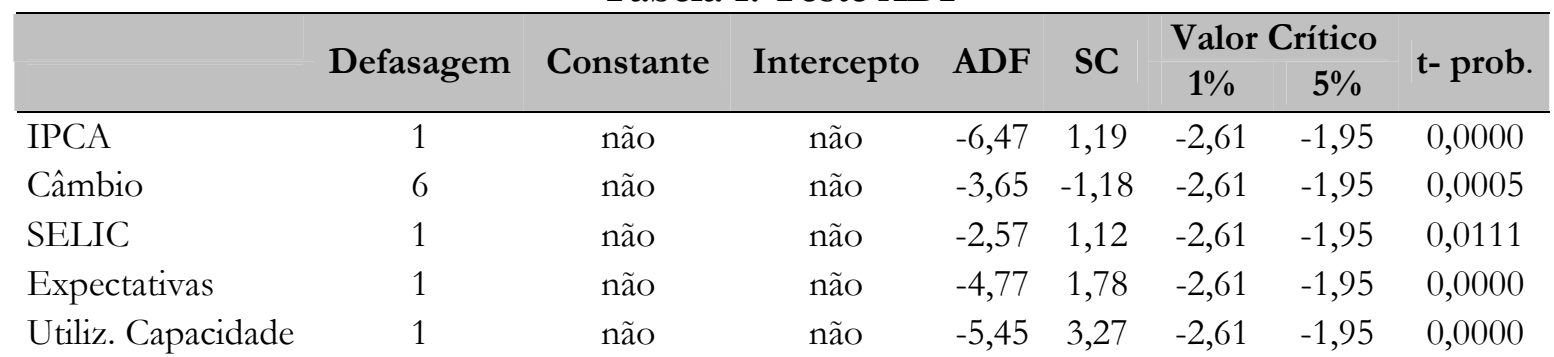

Fonte: elaborado a partir dos dados da pesquisa.

Tabela 2. Seleção da defasagem do modelo

\begin{tabular}{cccc}
\hline Defasagens & AIC $^{1}$ & SC $^{2}$ & HQ $^{\mathbf{3}}$ \\
\hline 0 & 12.50091 & 12.69395 & 12.57415 \\
1 & 5.118179 & 6.276436 & 5.557620 \\
2 & 3.498213 & 5.621685 & 4.303856 \\
3 & 3.044417 & 6.133103 & 4.216260 \\
4 & 2.474988 & 6.528889 & 4.013033 \\
5 & 1.827394 & 6.846509 & 3.731639 \\
6 & 1.315889 & 7.300218 & 3.586335 \\
7 & -0.507907 & 6.441637 & 2.128740 \\
$\mathbf{8}$ & $\mathbf{- 2 . 8 3 5 1 4 3 *}$ & $\mathbf{5 . 0 7 9 6 1 6 *}$ & $\mathbf{0 . 1 6 7 7 0 6}$ \\
\hline
\end{tabular}

Fonte: elaborado a partir dos dados da pesquisa.

Notas: 1) AIC: Akaike Information Criterion;

2) SC: Schwarz Criterion;

3) HQ: Hannan-Quinn Criterion.

${ }^{23}$ Para uma discussão sobre esse fator, ver Amaral e Oreiro (2006) e também Bacha e Chrysostomo (2006). 
Tabela 3. Teste LM de correlação serial dos resíduos

\begin{tabular}{ccc}
\hline Lags & LM-Stat & Prob. \\
\hline 1 & 32.77274 & 0.1368 \\
2 & 32.77698 & 0.1367 \\
3 & 34.47751 & 0.0981 \\
4 & 21.12635 & 0.6855 \\
5 & 41.13912 & 0.0222 \\
6 & 20.35691 & 0.7279 \\
7 & 21.04898 & 0.6899 \\
$\mathbf{8}$ & $\mathbf{2 0 . 6 8 5 9 3}$ & $\mathbf{0 . 7 1 0 0}$ \\
\hline
\end{tabular}

Fonte: elaborado a partir dos dados da pesquisa.

Tabela 4. Teste de Causalidade de Granger

\begin{tabular}{lcc}
\hline \multicolumn{1}{c}{ Hipótese Nula } & Estatística F & Probabilidade \\
\hline UTIL.CAP. does not Granger Cause SELIC & 0.49241 & 0.85243 \\
SELIC does not Granger Cause UTIL.CAP. & $\mathbf{2 . 3 7 9 9 0}$ & $\mathbf{0 . 0 3 8 9 1}$ \\
IPCA does not Granger Cause SELIC & $\mathbf{2 . 8 1 4 1 5}$ & $\mathbf{0 . 0 1 7 5 5}$ \\
SELIC does not Granger Cause IPCA & $\mathbf{3 . 1 6 9 0 8}$ & $\mathbf{0 . 0 0 9 2 7}$ \\
EXPEC. does not Granger Cause SELIC & $\mathbf{4 . 0 8 2 3 1}$ & $\mathbf{0 . 0 0 1 9 3}$ \\
SELIC does not Granger Cause EXPEC. & $\mathbf{2 . 0 1 0 3 6}$ & $\mathbf{0 . 0 7 7 1 6}$ \\
CAMBIO does not Granger Cause SELIC & $\mathbf{3 . 7 8 6 7 6}$ & $\mathbf{0 . 0 0 3 1 7}$ \\
SELIC does not Granger Cause CAMBIO & 1.86777 & 0.10045 \\
IPCA does not Granger Cause UTIL.CAP. & 2.08936 & 0.06665 \\
UTIL.CAP. does not Granger Cause IPCA & 0.21977 & 0.98481 \\
EXPEC. Does not Granger Cause UTIL.CAP. & 0.85034 & 0.56676 \\
UTIL.CAP. does not Granger Cause EXPEC. & 0.77025 & 0.63118 \\
CAMBIO does not Granger Cause UTIL.CAP. & 0.88868 & 0.53689 \\
UTIL.CAP. does not Granger Cause CAMBIO & 1.14836 & 0.35949 \\
EXPEC. does not Granger Cause IPCA & $\mathbf{5 . 3 2 3 5 3}$ & $\mathbf{0 . 0 0 0 2 7}$ \\
IPCA does not Granger Cause EXPEC. & $\mathbf{3 . 1 4 0 0 9}$ & $\mathbf{0 . 0 0 9 7 7}$ \\
CAMBIO does not Granger Cause IPCA & $\mathbf{5 . 8 8 8 9 6}$ & $\mathbf{0 . 0 0 0 1 2}$ \\
IPCA does not Granger Cause CAMBIO & 1.69277 & 0.13851 \\
CAMBIO does not Granger Cause EXPEC. & $\mathbf{6 . 7 2 6 1 3}$ & $\mathbf{3 . 7 E - 0 5}$ \\
EXPEC. Does not Granger Cause CAMBIO & 1.81067 & 0.11159 \\
\hline
\end{tabular}

Fonte: elaborado a partir dos dados da pesquisa. 
Tabela 5. Decomposição da Variância do Modelo Decomposição da Variância das Expectativas

\begin{tabular}{ccccc}
\hline Lag & Câmbio & IPCA & Selic & UTIL. CAP. \\
\hline 1 & 0.000000 & 0.000000 & 0.000000 & 0.000000 \\
2 & 13.57681 & 1.573058 & 2.400614 & 0.425770 \\
3 & 39.40960 & 2.944406 & 4.864823 & 1.179779 \\
4 & 52.53857 & 3.600536 & 5.747780 & 0.857362 \\
5 & 57.50934 & 11.03806 & 5.852558 & 0.628239 \\
6 & 62.89066 & 13.48834 & 6.035297 & 1.294459 \\
7 & 65.51888 & 13.31527 & 6.340326 & 2.907287 \\
8 & 65.06638 & 14.45176 & 5.758733 & 4.554007 \\
9 & 64.39078 & 14.87729 & 5.469809 & 5.577097 \\
10 & 64.27262 & 15.05454 & 5.369170 & 5.752989 \\
11 & 62.91333 & 15.05337 & 5.211922 & 5.657032 \\
12 & 60.19018 & 14.81885 & 4.971508 & 5.893091 \\
\hline
\end{tabular}

Fonte: elaborado a partir dos dados da pesquisa.

Decomposição da Variância do IPCA

\begin{tabular}{ccccc}
\hline Lag & EXPEC. & Câmbio & SELIC & UTIL. CAP. \\
\hline 1 & 42.81935 & 1.384802 & 0.000000 & 0.000000 \\
2 & 43.11917 & 27.53886 & 5.600814 & 1.074364 \\
3 & 36.37567 & 37.48427 & 4.709472 & 1.175454 \\
4 & 33.29919 & 36.97458 & 9.956265 & 1.567769 \\
5 & 28.44837 & 34.38752 & 7.036630 & 1.782348 \\
6 & 24.59696 & 41.36088 & 8.092413 & 2.340364 \\
7 & 20.70507 & 44.41125 & 7.548838 & 3.780822 \\
8 & 18.92537 & 45.78073 & 6.910271 & 4.142679 \\
9 & 19.60365 & 44.67476 & 6.980214 & 4.871283 \\
10 & 19.54470 & 44.86808 & 6.952595 & 5.014848 \\
11 & 24.40846 & 42.21354 & 6.476920 & 4.789022 \\
12 & 27.20401 & 40.47260 & 6.128907 & 5.138000 \\
\hline
\end{tabular}

Fonte: elaborado a partir dos dados da pesquisa.

Decomposição da variância da Selic

\begin{tabular}{ccccc}
\hline Lag & EXPEC. & Câmbio & IPCA & UTIL. CAP. \\
\hline 1 & 0.076636 & 10.16066 & 1.890519 & 0.000000 \\
2 & 13.43011 & 7.468748 & 0.568916 & 0.525938 \\
3 & 25.03182 & 5.326136 & 1.124728 & 1.057065 \\
4 & 23.44855 & 7.854803 & 3.041997 & 0.890784 \\
5 & 18.93049 & 16.77753 & 6.213429 & 0.634196 \\
6 & 12.86437 & 32.82618 & 10.58210 & 0.445535 \\
7 & 8.141439 & 43.71657 & 16.29865 & 0.687722 \\
8 & 5.688416 & 49.64955 & 17.98029 & 1.686585 \\
9 & 4.364261 & 53.70251 & 17.93784 & 3.024596 \\
10 & 3.667128 & 55.89113 & 17.65213 & 3.811567 \\
11 & 3.254811 & 56.79474 & 18.06610 & 4.130433 \\
12 & 3.018289 & 56.97770 & 19.00996 & 4.140307 \\
\hline
\end{tabular}

Fonte: elaborado a partir dos dados da pesquisa. 
Decomposição da variância da utilização capacidade

\begin{tabular}{ccccc}
\hline Lag & EXPEC. & Câmbio & IPCA & SELIC \\
\hline 1 & 6.691998 & 16.96313 & 0.548012 & $\mathbf{0 . 2 3 5 1 6 5}$ \\
2 & 26.32707 & 15.00224 & 3.307147 & $\mathbf{4 . 2 4 2 5 8 3}$ \\
3 & 28.39903 & 12.56459 & 8.568046 & $\mathbf{1 3 . 9 6 0 2 0}$ \\
4 & 39.44393 & 10.38081 & 6.942341 & $\mathbf{1 2 . 3 5 2 6 2}$ \\
5 & 38.31649 & 10.54187 & 7.967644 & $\mathbf{1 3 . 0 5 5 8 8}$ \\
6 & 37.72009 & 10.40986 & 8.068921 & $\mathbf{1 2 . 9 5 7 6 7}$ \\
7 & 35.05529 & 14.36501 & 7.482912 & $\mathbf{1 3 . 5 9 9 8 0}$ \\
8 & 31.61594 & 17.50996 & 9.443820 & $\mathbf{1 4 . 5 1 4 1 2}$ \\
9 & 28.46092 & 15.74149 & 9.629252 & $\mathbf{2 0 . 6 6 1 4 5}$ \\
10 & 26.77223 & 15.05201 & 10.45574 & $\mathbf{2 0 . 1 1 0 2 0}$ \\
11 & 26.69893 & 14.89203 & 11.04907 & $\mathbf{1 9 . 9 3 2 3 0}$ \\
12 & 26.72581 & 14.80472 & 11.23484 & $\mathbf{1 9 . 9 4 6 5 9}$ \\
\hline
\end{tabular}

Fonte: elaborado a partir dos dados da pesquisa.

Tabela 6. Teste de Cointegração de Johansen

\begin{tabular}{|c|c|c|c|}
\hline \multirow{2}{*}{$\begin{array}{c}\text { Hipótese de } \\
\text { não } \\
\text { cointegração }\end{array}$} & Traço & $5 \%$ & $1 \%$ \\
\hline & Estatística & Valor Crítico & Valor Crítico \\
\hline Nenhum ** & 134.8688 & 59.46 & 66.52 \\
\hline Ao menos $1 * *$ & 62.87330 & 39.89 & 45.58 \\
\hline Ao menos $2 * *$ & 16.60687 & 24.31 & 29.75 \\
\hline Ao menos $3 * *$ & 2.244890 & 12.53 & 16.31 \\
\hline Ao menos $4 * *$ & 0.397960 & 3.84 & 6.51 \\
\hline
\end{tabular}

Fonte: elaborado a partir dos dados da pesquisa.

(**) Denota a rejeição da hipótese nula a 5\% e 1\%.

O Traço indica pelo menos 1 vetor de co-integração ao nível de $1 \%$.

Tabela 7. Teste de Endogeneidade das Variáveis

(VAR Pairwise Granger Causality/Block Exogeneity Wald Tests)

\begin{tabular}{|c|c|c|c|c|c|c|c|c|c|c|}
\hline \multirow{3}{*}{ Variáveis } & \multicolumn{10}{|c|}{ Variável Dependente } \\
\hline & \multicolumn{2}{|c|}{ EXPEC. } & \multicolumn{2}{|c|}{ IPCA } & \multicolumn{2}{|c|}{ SELIC } & \multicolumn{2}{|c|}{ UTIL. CAP. } & \multicolumn{2}{|c|}{ CÂMBIO } \\
\hline & Chi-sq & Prob. & Chi-sq & Prob. & Chi-sq & Prob & Chi-sq & Prob & Chi-sq & Prob \\
\hline EXPEC. & - & - & 24.42 & 0.00 & 11.66 & 0.06 & 5.67 & 0.46 & 17.93 & 0.00 \\
\hline Câmbio & 32.33 & 0.0000 & 19.03 & 0.00 & 4.82 & 0.56 & 4.89 & 0.55 & - & - \\
\hline IPCA & 6.32 & 0.3880 & - & - & 10.77 & 0.09 & 12.29 & 0.05 & 7.80 & 0.25 \\
\hline SELIC & 5.13 & 0.5270 & 9.52 & 0.14 & - & - & 10.38 & 0.10 & 14.68 & 0.02 \\
\hline $\begin{array}{l}\text { UTIL. } \\
\text { CAP }\end{array}$ & 4.60 & 0.5957 & 2.59 & 0.85 & 6.87 & 0.33 & - & - & 3.01 & 0.80 \\
\hline
\end{tabular}

Fonte: elaborado a partir dos dados da pesquisa.

Nota: A relação de exogeneidade é a seguinte: taxa de câmbio, expectativas, IPCA, Selic e utilização da capacidade. 
Figura 2. Funções Impulso Resposta

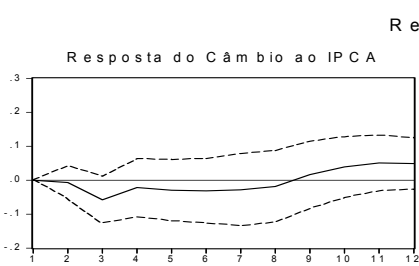

Response to Cholesky One S.D. Innovations \pm 2 S.E.
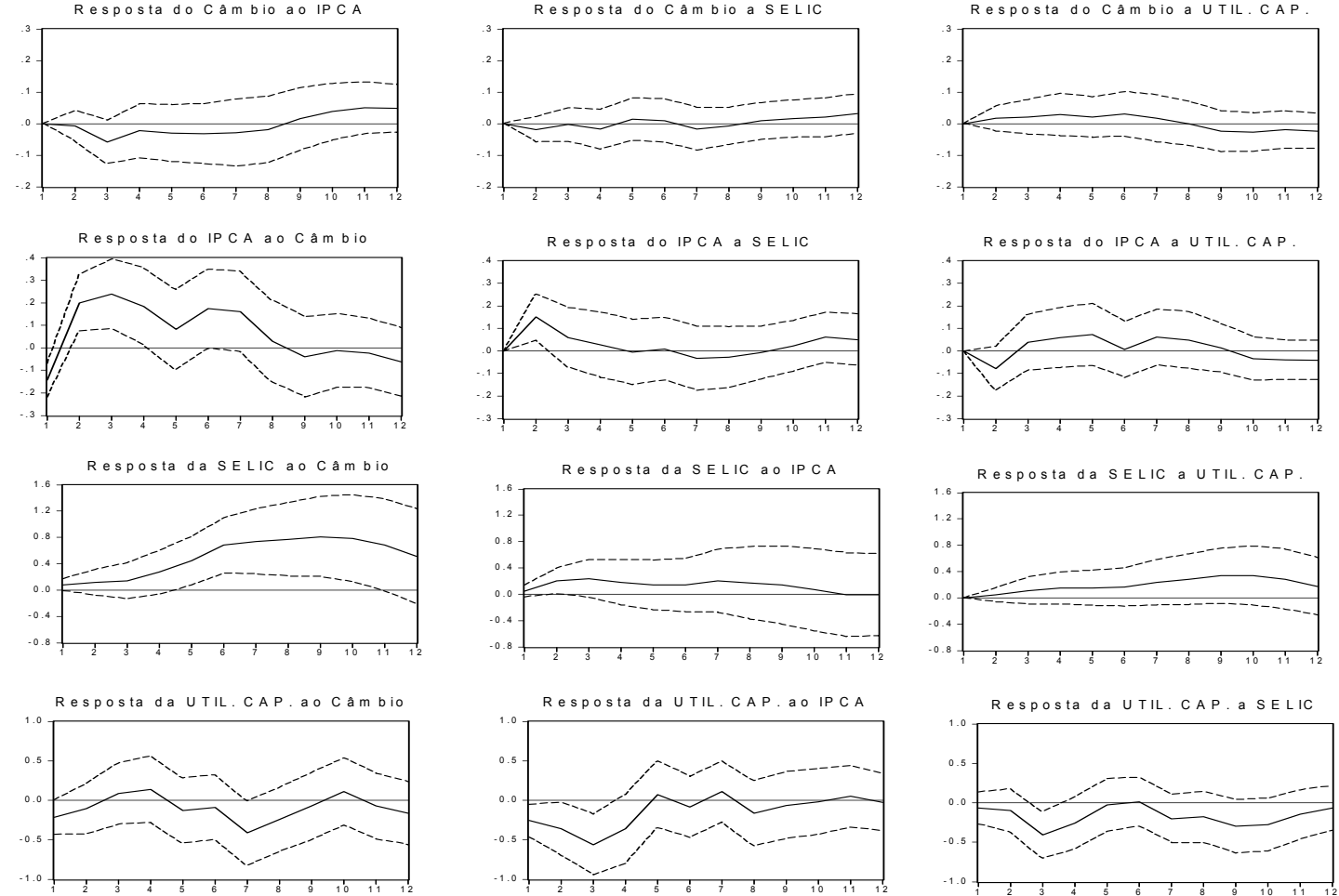

Fonte: elaborado a partir dos dados da pesquisa.

\section{Referências Bibliográficas}

AMARAL, R. Q.; MOTTA, F. C. Uma análise da política monetária brasileira: a determinação da inflação e a Regra de Taylor do Banco Central. In: Encontro Nacional de Economia, ANPEC-SUL. Florianópolis, 2006.

AMARAL, R. Q.; OREIRO, J. L. Armadilha do regime de politica monetária brasileiro: a existência de equilíbrios múltiplos. In: Encontro nacional de economia política, SEP, XI. Vitória, 2006.

ASTERIOUS, D. Applied Econometrics: a modern approach using eviews and microfit. New York, Palgrave Mcmillan, 2006, 423 p.

BACHA, E. L.; OLIVEIRA, L. C. Mercado de capitais e dívida pública. Rio de Janeiro: Contra Capa, 2006.

BANCO CENTRAL DO BRASIL. Banco de dados estatísticos: Disponível em: www.bacen.gov.br. Acesso em: jul. 2006.

ENDERS, W. Applied economic time series, Wiley Press, 1995

GRANGER, C. W. J. (1969). Investigating causal relations by econometric models and crossspectral methods. Econometrica, Cambridge, MIT, 37. p. 99-126.

GREENE, W. H. Econometric Analysis. Pearson Education: Delhi, 2005, 1026 p.

IPEA. Banco de dados estatísticos. Disponível em: www.ipea.gov.br. Acesso em: jul. 2006.

PINDYCK, R. S.; RUBINFELD, D. L. Econometric models and economic forecasts. New York: Mc Graw-Hill, 1991.596 p.

RELATÓRIO DE INFLAÇÃO. Banco Central do Brasil, jun. 1999 a mar. 2006 (publicação trimestral). Disponível em: www.bcb.gov.br/?RELINF 
